\title{
Heartbeat: prevention of bullying in medical training by improving the work environment
}

doi:10.1136/heartjnl-2021-320732

Although the experience of bullying is subjective, there is increasing evidence that bullying during physician training is associated with an increased risk of serious medical errors as well as negatively impacting job satisfaction and the likelihood of remaining in fulltime medical practice. In a survey of 1358 cardiology trainees between 2017 and 2020, Camm and colleagues ${ }^{1}$ found that bullying was reported by $11 \%$ overall. Compared with men, women were more likely to be bullied (OR: $1.5595 \%$ CI 1.08 to 2.21) and to report sexist language ( $14 \%$ vs $4 \%, \mathrm{p}<0.001)$. Graduates from medical schools outside the UK, including those from the European Economic Area (EEA) schools) also were more likely to be bullied and to experience racist language (UK 1.5\%, EEA $6 \%$, other locations $7 \%, \mathrm{p}=0.006)$. The most common job roles of those reported to be bullying included cardiology and other consultants, other medical staff and non-medical staff, but only rarely other trainees. An even larger issue is that $33 \%$ of trainees experienced inappropriate behaviour (figure 1), even when not reported as bullying.

In the accompanying editorial, Baruah and Sedgwick ${ }^{2}$ discuss approaches to eliminating bullying which include 'focusing on improvements in seemingly tangential issues, such as wider work-life balance, remuneration, working conditions and workload, which may act to improve workplace culture and prevent the behaviours occurring in the first place, making a better working environment for all.' In addition, we need to create behaviour toolkits, workshops and behaviour champions. 'Both perpetrators and victims need to be involved and supported in order to bring about organisational behavioural change through reflection, counselling, training and coaching, with an avoidance of

Division of Cardiology, University of Washington, Seattle, Washington, USA

Correspondence to Professor Catherine M Otto, Division of Cardiology, University of Washington, Seattle, WA 98195, USA; cmotto@uw.edu

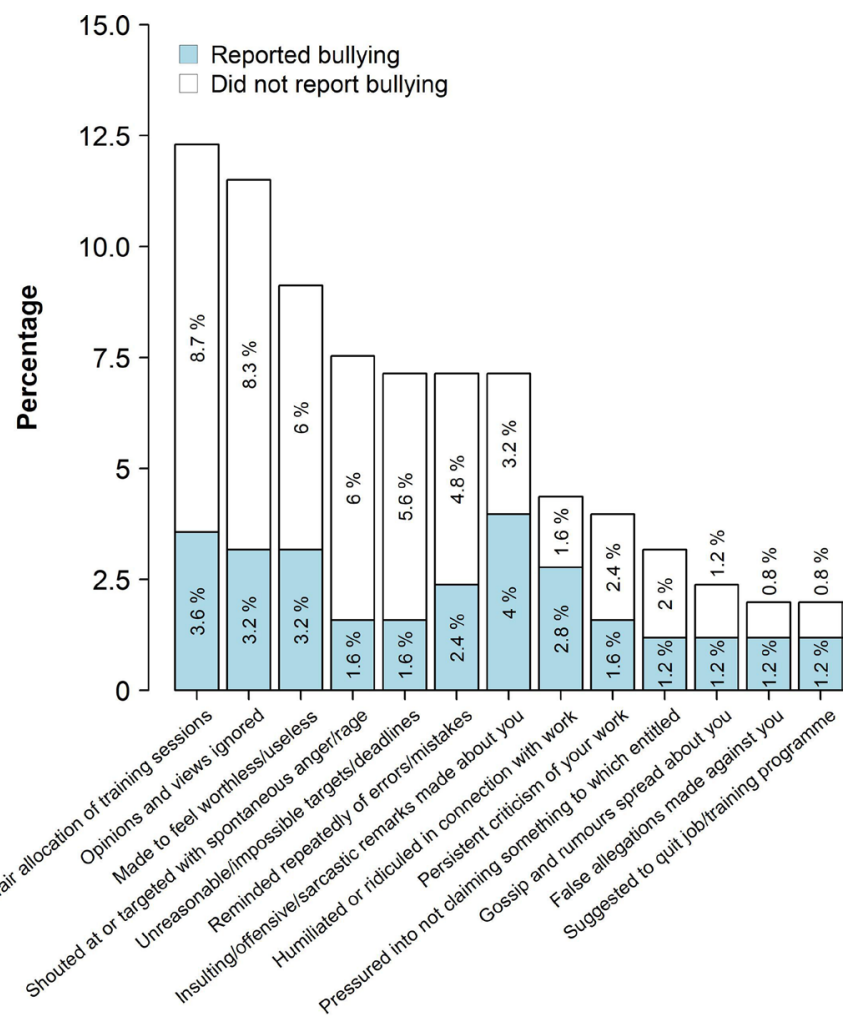

Inappropriate behaviour experienced

Figure 1 Bar plot demonstrating inappropriate behaviour reported by cardiology trainees divided into those who reported bullying (blue) and those who did not (white). Bars represent the percentage of trainees reporting inappropriate behaviour. participants limited to those completing the survey in $2020(n=252)$.

placing too much onus on the 'victim' and their supposed resilience.'

In order to better define the role of coronary fractional flow reserve calculated by CT imaging $\left(\mathrm{FFR}_{\mathrm{CT}}\right)$ for prediction of prognosis in patients with stable coronary artery disease (CAD), Nørgaard and colleagues $^{3}$ performed a systematic review and meta-analysis with a primary endpoint of all-cause mortality or myocardial infraction over a 12 month follow-up period. An $\mathrm{FFR}_{\mathrm{CT}}>0.80$ identified a higher risk group with the primary endpoints occurring in $1.4 \%$ (47/3334) compared with only $0.6 \%$ $(13 / 2126)$ of those with FFR $_{\mathrm{CT}} \leq 0.80$ (relative risk (RR) 2.31 (95\% CI 1.29 to 4.13 ), $\mathrm{p}=0.005$ ) (figure 2). There was a continuous inverse relationship between FFR $_{\mathrm{CT}}$ and the risk of adverse events with each 0.10 -unit $\mathrm{FFR}_{\mathrm{CT}}$ reduction associated with a greater risk of the primary endpoint (RR 1.67 (95\% CI 1.47 to 1.87$), \mathrm{p}<0.001)$.

Williams and $\mathrm{Newby}^{4}$ discuss the ability of coronary CT angiography (CCTA) to measure stenosis severity, visualise plaque and determine $\mathrm{FFR}_{\mathrm{CT}}$ (figure 3). They raise 'the question of what is driving the association between $\mathrm{FFR}_{\mathrm{CT}}$ and clinical outcome. Is it the ischaemic burden measured by the fractional flow reserve or is it mediated through the association of fractional flow reserve with adverse plaque characteristics?' Either way, 'FFR is only one of the many measures that CCTA can provide and other variables, such as quantitative plaque assessment, are emerging as important prognostic indicators. We now need to identify which are the best to use for diagnosis, risk stratification and treatment decisions to enable 


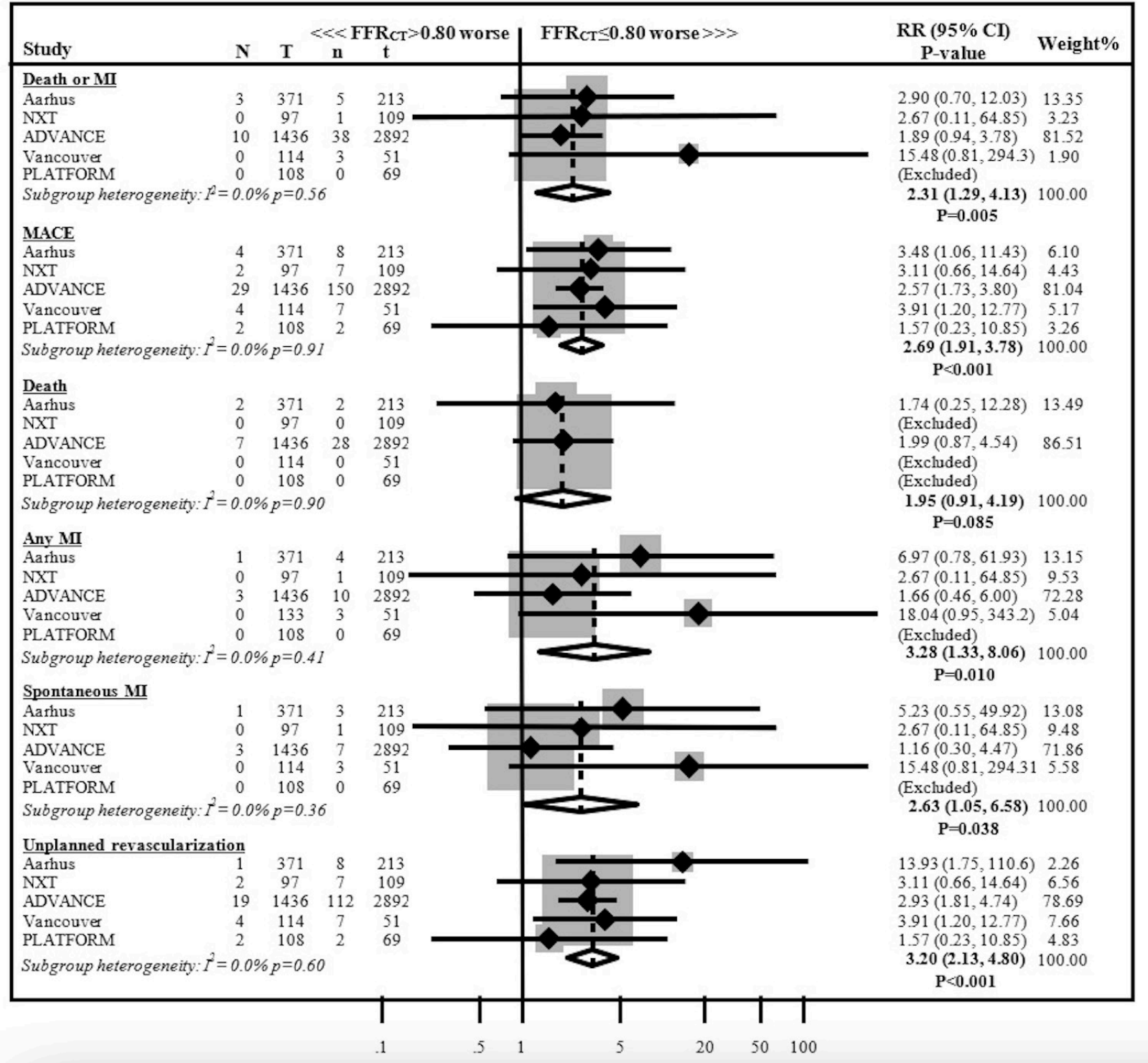

Figure 2 Meta-analysis of the primary composite endpoint (death or any MI) and secondary endpoints at 12 month follow-up. FFR $>0.80$ : $\mathrm{N}=$ number of patients with adverse events; $\mathrm{T}=$ total number of patients. $\mathrm{FFR}_{\mathrm{CT}} \leq 0.80$ : $\mathrm{N}$ and $\mathrm{t}=$ number of patients with adverse events and total number of patients. Strata with zero events were not included in the analysis. mace (major adverse cardiac event) was defined as a composite of death, any MI or unplanned revascularisation. unplanned revascularisation was defined as any revascularisation (percutaneous coronary intervention and/or coronary artery bypass grafting) occurring between 3 month and 12 month follow-up. ADVANCE, assessing diagnostic value of non-invasive

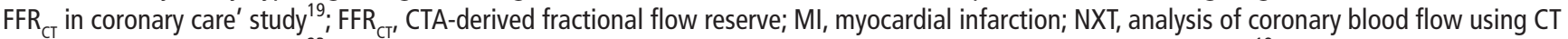
angiography: next steps trial ${ }^{23}$; PLATFORM, prospective longitudinal trial of $\mathrm{FFR}_{\mathrm{CT}}$ : outcome and resource impacts trial ${ }^{18} ; \mathrm{RR}$, risk ratio.

the optimal management and outcomes for our patients.'

Another interesting paper in this issue of Heart reports hospital re-admission rates after transcatheter aortic valve implantation (TAVI) based on a database that included almost 45 thousand TAVI procedures. ${ }^{5}$ Although the median 30-day re-admission rate was $11.8 \%$, there was wide variation between hospitals related to patient, hospital and economic factors. Further understanding of the factors leading to this variance might result in lower re-admission rates.
A review article in this issue summarises the association between preterm birth and the lifetime risk of ischaemic heart disease and heart failure in the context of a higher prevalence of cardiovascular risk factors that include hypertension, metabolic syndrome and diabetes ${ }^{6}$ (figure 4).

The Education in Heart article in this issue $^{7}$ provides the basic principles for implantable left ventricular assist devices including indications, eligibility and current outcomes. Key messages are:

- "Continuous-flow left ventricular assist devices (LVADs) are an established treatment for carefully selected patients with advanced heart failure, with superior survival to those managed on medical therapy alone.

- The majority of patients supported on LVAD have significantly improved quality of life and increased functional status following implantation.

- Although 2year survival following LVAD implantation is now similar to that following cardiac transplantation, medium-term to longer-term survival remains superior in those undergoing transplantation. 


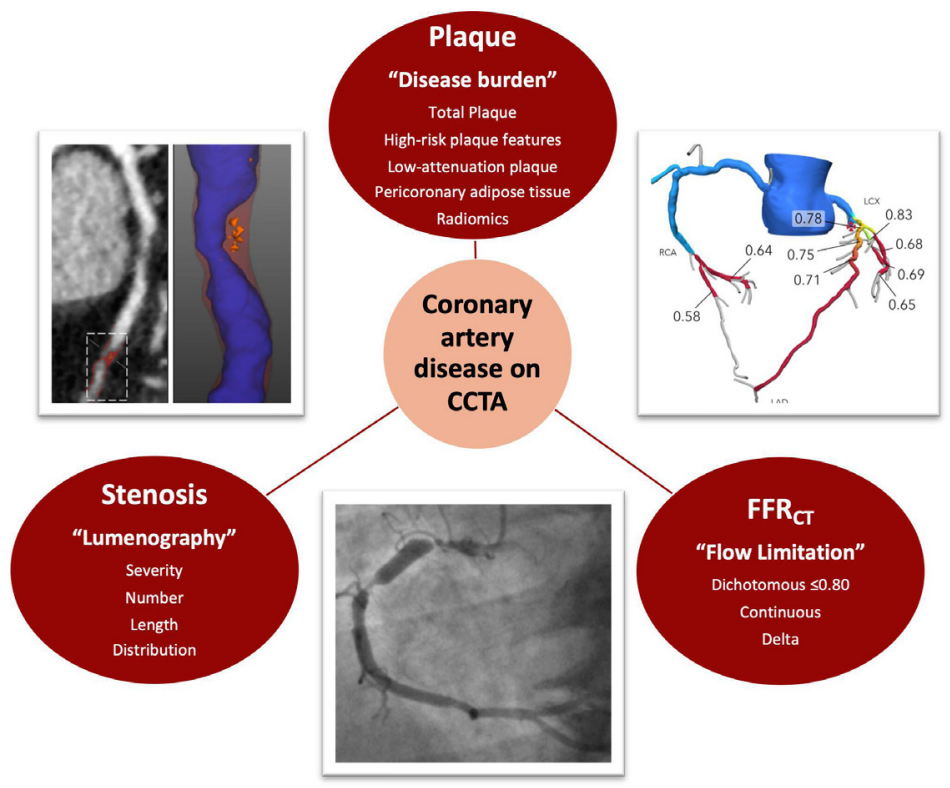

Figure 3 Overlap between coronary CT angiography (CCTA) parameters in coronary artery disease.

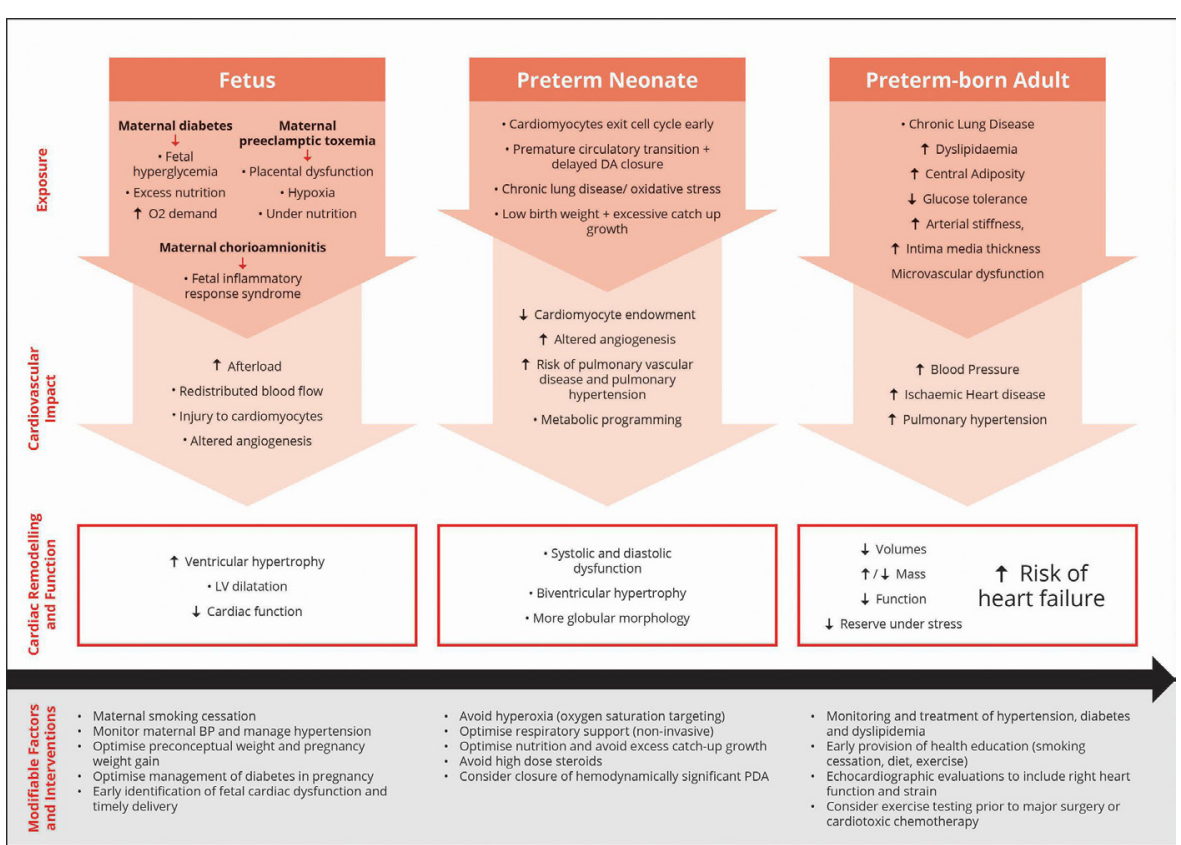

Figure 4 Exposures and mechanisms for altered cardiac structure and function in young adults born preterm. BP, Blood pressure; DA, ductus arteriosus; LV, left ventricle.
- Infection, bleeding and neurological events remain the predominant adverse events after implant.

- Reduction in readmissions and adverse event rates is necessary for LVADs to become cost-effective and a viable longer-term alternative to cardiac transplantation.”

Funding The authors have not declared a specific grant for this research from any funding agency in the public, commercial or not-for-profit sectors.

Competing interests None declared.

Patient and public involvement Patients and/or the public were not involved in the design, or conduct, or reporting, or dissemination plans of this research.

Patient consent for publication Not applicable.

Ethics approval This study does not involve human participants.

Provenance and peer review Commissioned; internally peer reviewed.

(C) Author(s) (or their employer(s)) 2022. No commercial re-use. See rights and permissions. Published by BMJ.

\section{A) Check for updates}

To cite Otto CM. Heart 2022;108:157-159.

Heart 2022;108:157-159.

doi:10.1136/heartjnl-2021-320732

ORCID iD

Catherine M Otto http://orcid.org/0000-0002-05279392

\section{REFERENCES}

1 Camm CF, Joshi A, Moore A, et al. Bullying in UK cardiology: a systemic problem requiring systemic solutions. Heart 2022;108:212-8.

2 Baruah R, Sedgwick E. Bullying in UK cardiology: a systemic problem requiring systemic solutions. Heart 2022;108:162-3.

3 Nørgaard BL, Gaur S, Fairbairn TA, et al. Prognostic value of coronary computed tomography angiographic derived fractional flow reserve: a systematic review and meta-analysis. Heart 2022;108:194-202.

4 Williams MC, Newby DE. Prognostic value of fractional flow reserve from computed tomography. Heart 2022;108:160-1

5 Ando T, Ashraf S, Kuno T, et al. Hospital variation of 30-day readmission rate following transcatheter aortic valve implantation. Heart 2022;108:219-24.

6 Greer C, Troughton RW, Adamson PD, et al. Preterm birth and cardiac function in adulthood. Heart 2022;108:172-7.

7 Bhagra SK, Pettit S, Parameshwar J. Implantable left ventricular assist device: indications, eligibility and current outcomes. Heart 2022;108:233-41. 